\title{
Bourdons rares du Parc Naturel Régional du Queyras (Hautes-Alpes, France)
}

\section{Par Gilles MAHÉ *}

\begin{abstract}
The author, who stayed in the Parc Naturel Régional du Queyras (France) between the $9^{\text {th }}$ and the $17^{\text {th }}$ August 2007, noted the presence of more than twenty species of bumblebees. This paper deals with the most interesting observations about Bombus gerstaeckeri MORAWITZ, B. inexspectatus (TKALCU), B. brodmannicus VOGT and the copulation of B. mendax GERSTAECKER.
\end{abstract}

Résumé. L'auteur, qui a séjourné dans le Parc Naturel Régional du Queyras (France) entre le 9 et le 17 août 2007, y a noté la présence de plus d'une vingtaine d'espèces de bourdons. Cet article rapporte les observations les plus intéressantes concernant Bombus gerstaeckeri MORAWITZ, B. inexspectatus (TKALCU), B. brodmannicus VOGT et l'accouplement de B. mendax GERSTAEGKER.

Mots-clés. Parc Naturel Régional du Queyras, Bombus gerstaeckeri, Bombus inexspectatus, Bombus brodmannicus, Bombus mendax, Aconitum anthora, Aconitum napellus, Aconitum vulparia, Delphinium dubium.

\section{Bombus gerstaeckeri dans le Queyras (France)}

En 2006 j'avais noté la présence de Bombus gerstaeckeri, bourdon spécialisé sur les aconits, en plusieurs localités du Parc Naturel National des Ecrins (Mahé 2007). En 2007 j'ai constaté que $B$. gerstaeckeri était également bien présent dans le Parc Naturel Régional du Queyras là où se trouvent des stations d'aconits. Je l'ai trouvé le 11 août sur Aconitum anthora L. (Figure 1) dans la vallée du Guil à la Cassine (Figure 2) (Hautes-Alpes, Ristolas ; WGS84 $\left.44,753^{\circ} \mathrm{N} 6,983^{\circ} \mathrm{E} ; 1740 \mathrm{~m}\right)$, le 13 août sur $A$. vulparia Reichenb. et Delphinium dubium (ROUY \& FOUC.) PAWL. dans la vallée de l'Aigue blanche (Hautes-Alpes, Saint-Véran ; WGS84 44,674 N 6,899 $\left.{ }^{\circ} \mathrm{E} ; 2140 \mathrm{~m}\right)$, le 14 août sur $A$. vulparia, $A$. napellus L. et $D$. dubium en bordure du torrent de Bouchouse (Hautes-Alpes, Ristolas ; WGS84 44,737 N 6,993 ${ }^{\circ} \mathrm{E} ; 2100 \mathrm{~m}$ ), et enfin le 15 août sur $A$. vulparia et $D$. dubium dans la vallée du torrent de Peynin au niveau du ravin de Roche Rousse (Hautes-Alpes, Aiguilles ; WGS84 44,753으 $6,889^{\circ} \mathrm{E} ; 2000 \mathrm{~m}$ ) ainsi qu'au niveau du Vallon de Rasis (Hautes-Alpes, Aiguilles ; WGS84 44,748 ${ }^{\circ} \mathrm{N}$ $\left.6,897^{\circ} \mathrm{E} ; 2210 \mathrm{~m}\right)$.

\section{La pollinisation des Aconits et Dauphinelles}

C'est dans le Queyras que la Dauphinelle du Dauphiné, Delphinium dubium (ROUY \& FOUG.) PAWL., plante rare endémique des Alpes, est la plus répandue. Pour tenter de comprendre comment cette plante est pollinisée, j'ai comparé la structure de sa fleur à celle des aconits et j'ai observé les bourdons à l'œuvre, apportant ainsi quelques éléments de réponse aux questions que je m'étais posées l'an dernier dans le massif des Ecrins (Mahé 2007). Plusieurs études (Løken 1950, Rousseau 1994, Utelli \& Roy 2000) décrivent la

* Gilles Mahé, Rue de la matte 18, F-44600 Saint-Nazaire, France.

E-mail: gilles.mahe@wanadoo.fr

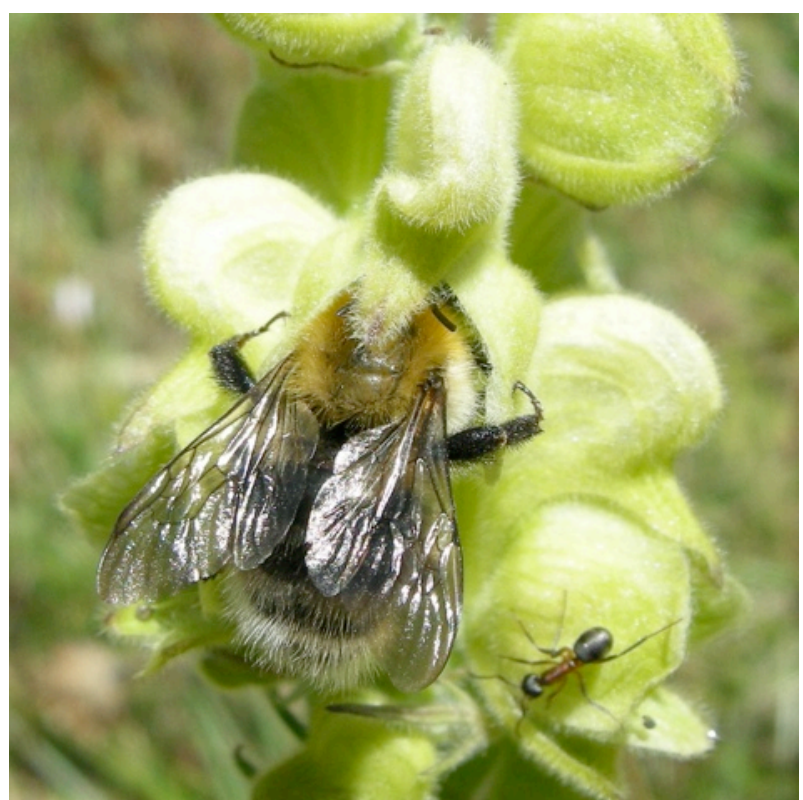

Figure 1. Bombus gerstaeckeri MORAWITZ sur Aconitum anthora (Ranunculaceae) (Photo G Mahé)

pollinisation des espèces du genre Aconitum. Chez $A$. anthora comme chez $A$. napellus (Figure 3 ) les étamines sont bien exposées et le pollen est libéré avant que les stigmates de la fleur soient matures. A un stade plus avancé de la floraison, les étamines se recroquevillent laissant alors apparaître nettement le pistil au centre de la fleur. Sur A. anthora j'ai noté qu'Apis mellifera butinait uniquement le pollen (Figure 4) alors que les femelles de bourdons (B. gerstaeckeri, B. wurflenii, B. hortorum, B. lucorum, B. sichelii et B. mesomelas) butinaient également le nectar. Les mâles de $B$. hortorum m'ont semblé être d'excellents pollinisateurs de ces deux aconits. La disposition des nectaires (Figures 5 \& 6) est telle qu'en butinant le nectar ils s'appliquent de grandes quantités de pollen à la base des pattes et au niveau des premiers sternites. 


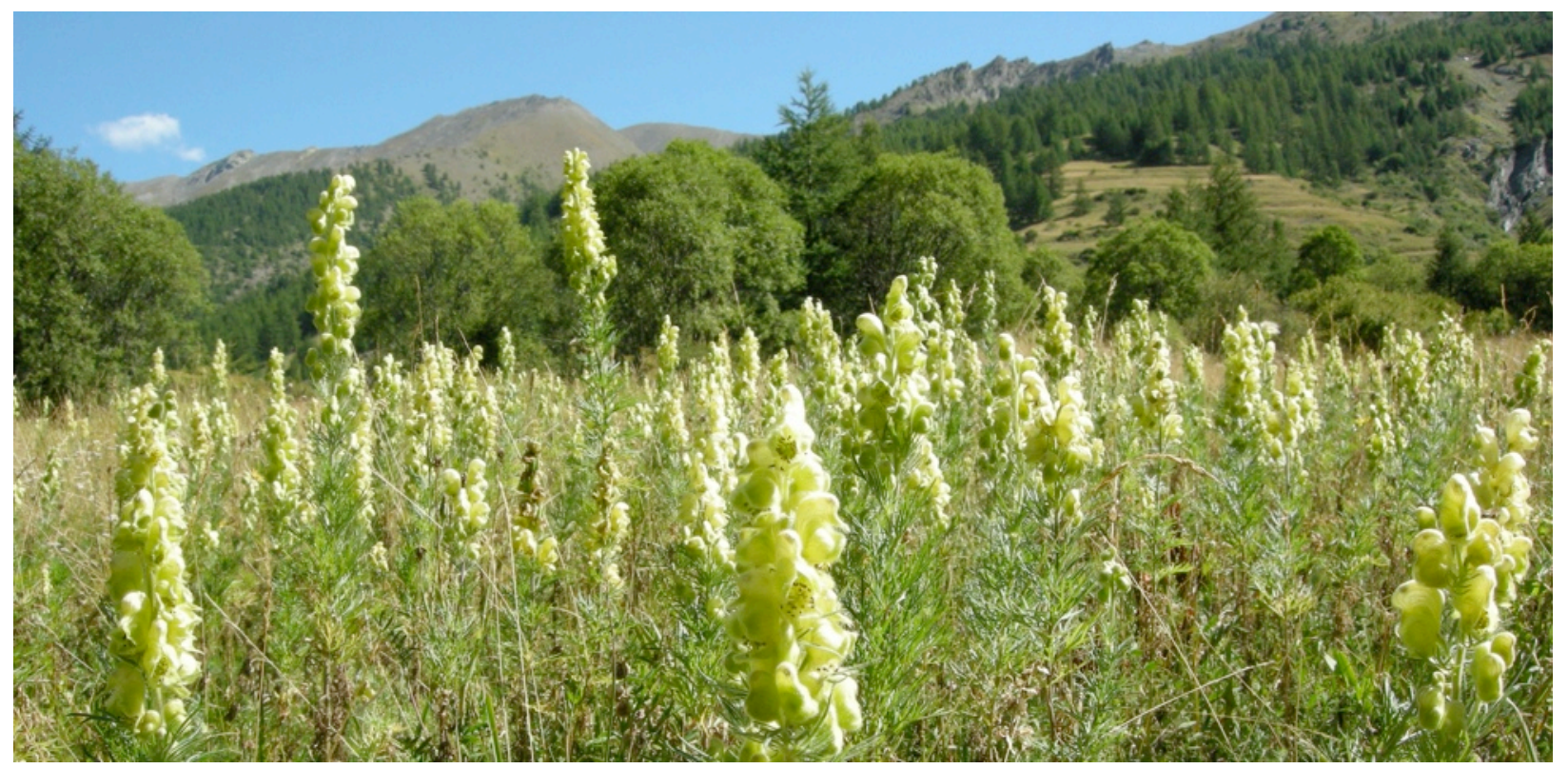

Figure 2. Le site de La Cassine (Hautes-Alpes, Ristolas, France) (Photo G Mahé)

La forme plus resserrée et allongée de la fleur d'A. vulparia ainsi que la disposition de l'éperon nectarifère (Figure 7) rend plus compliqué l'accès au nectar par la voie "normale". Seuls des bourdons à langue très longue comme $B$. hortorum ou B. gerstaeckeri y parviennent. B. wurflenii qui a la langue plus courte pratique un trou dans le casque juste au niveau de l'entrée de l'éperon (Figure 7) pour "voler" le nectar sans contribuer à la pollinisation de la fleur.

Chez D. dubium d'une part les étamines sont dissimulées derrière les pétales habituellement noirs ou exceptionnellement blancs (Figure 8), et d'autre part l'éperon nectarifère est droit. Cependant, comme chez les aconits, les étamines sont matures en premier, un certain nombre d'entre-elles étant d'ailleurs redressées (Figure 9). Lorsque les étamines fanent, apparaissent alors les styles du pistil également redressés (Figure 10). Ainsi lorsqu'un butineur de nectar enfonce sa langue au

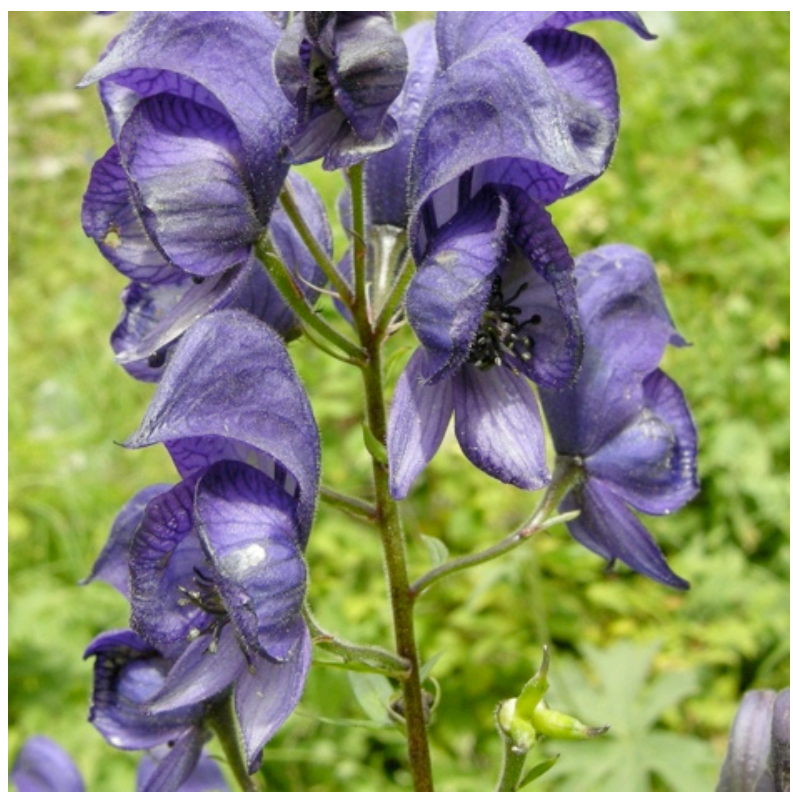

Figure 3. Détail d'une inflorescence d'Aconitum napellus (Ranunculaceae) (Photo G Mahé) fond de l'éperon, la base de son proboscis est appliquée entre les pétales qui s'écartent, contre les étamines ou les stigmates redressés suivant l'état de floraison de la fleur. Alors qu'en 2006 il m'avait semblé que les bourdons prélevaient du nectar sans toucher au pollen, j'ai pu vérifier cette année que les mâles et les femelles de $B$. gerstaeckeri et $B$. hortorum qui butinaient le nectar de D. dubium avaient systématiquement la base de leur proboscis chargée de pollen (Figure 11 ).

\section{Bombus inexspectatus}

Le site de la Cassine avec ses superbes stations d'A. anthora L. (Figure 2) offre une grande diversité d'autres fleurs. Sur Stachys recta L. j’ai capturé B. pyrenaeus, $B$. soroeensis et $B$. ruderarius, et sur Epilobium augustifolium, $B$. jonellus et B. hypnorum. C'est sur Centaurea sp. (Figure 12) que j'ai capturé une femelle et au moins trois mâles de

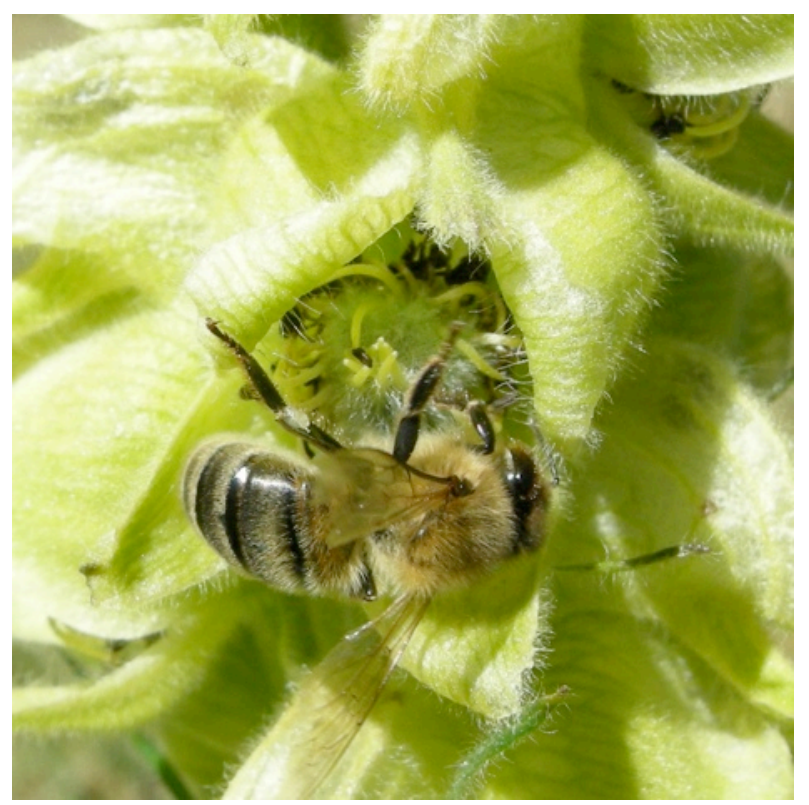

Figure 4. Une ouvrière d'Apis mellifera L. (Hymenoptera, Apidae) visitant Aconitum anthora (Photo G Mahé)

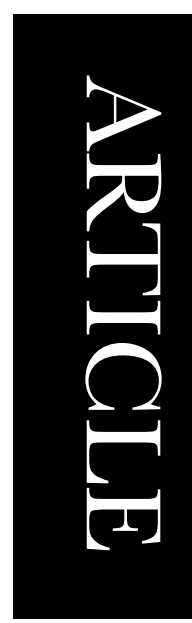




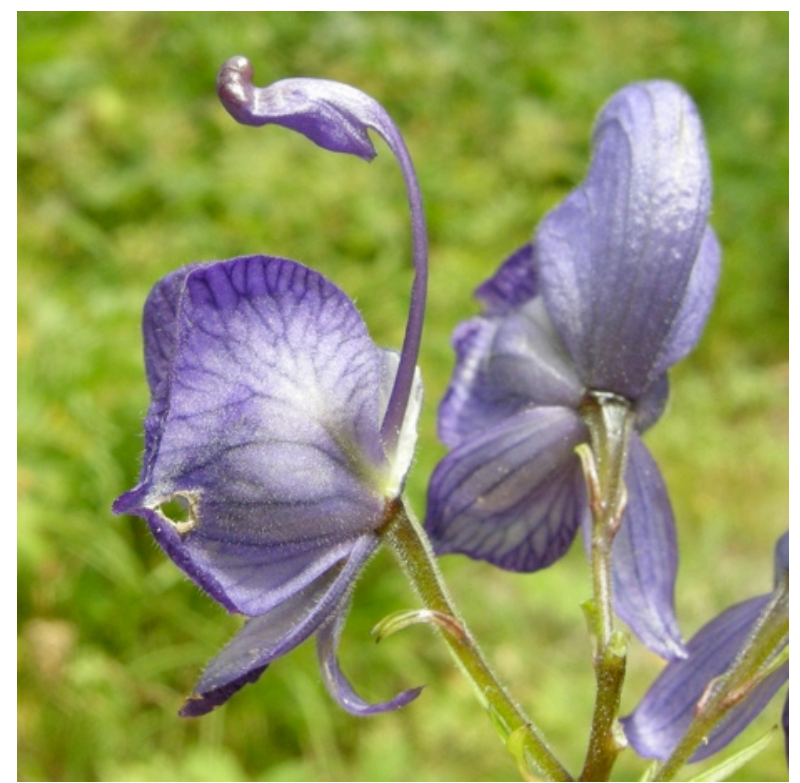

Figure 5. Disposition de l'éperon nectarifère chez Aconitum napellus (Ranunculaceae) (Photo G Mahé)

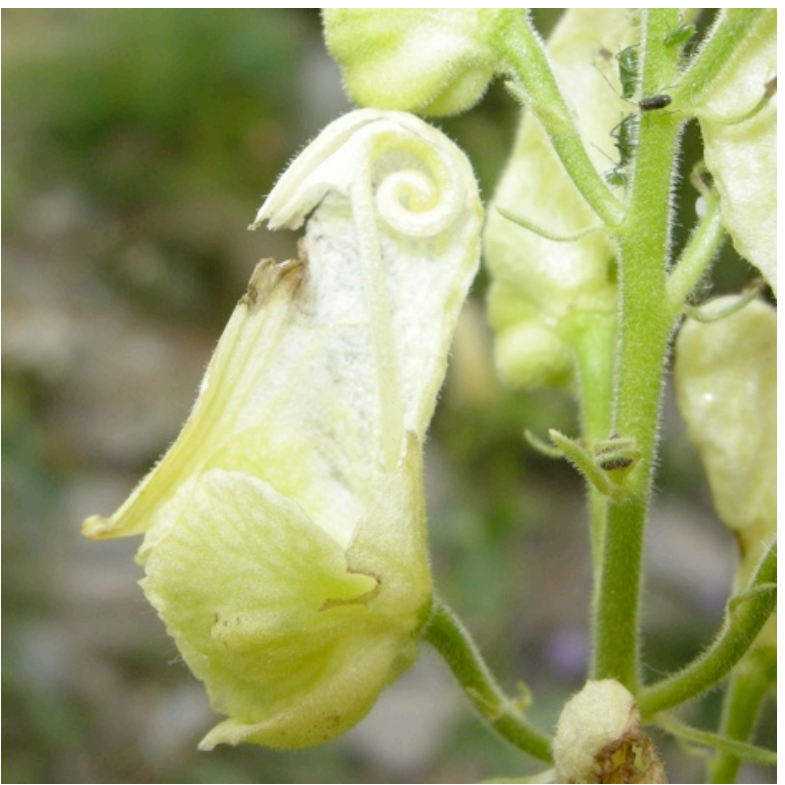

Figure 7. Disposition de l'éperon nectarifère chez Aconitum vulparia (Ranunculaceae) (Photo G Mahé)

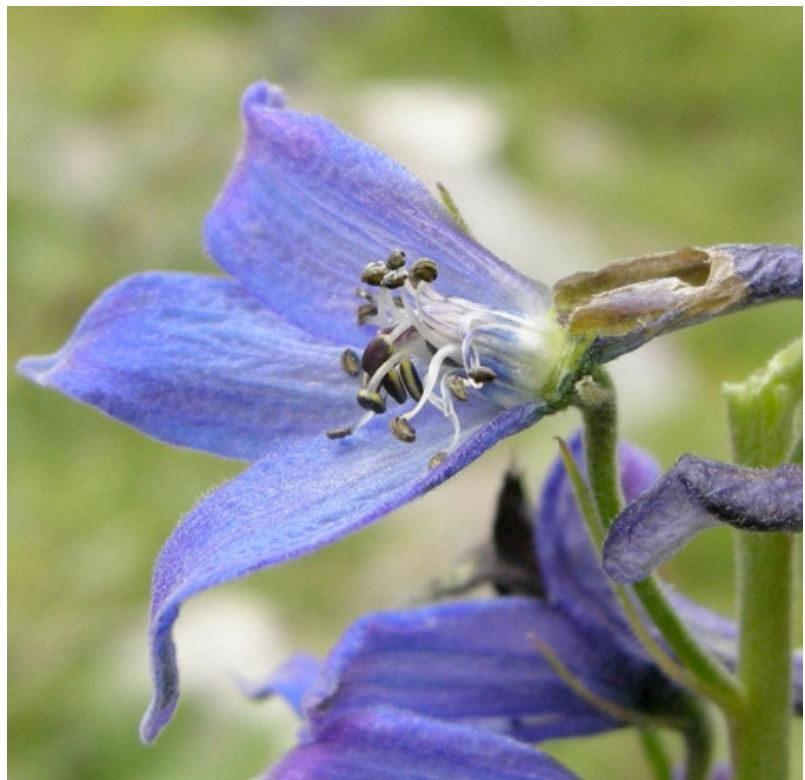

Figure 9. Redressement des étamines chez Delphinium dubium (Ranunculaceae) (Photo G Mahé)

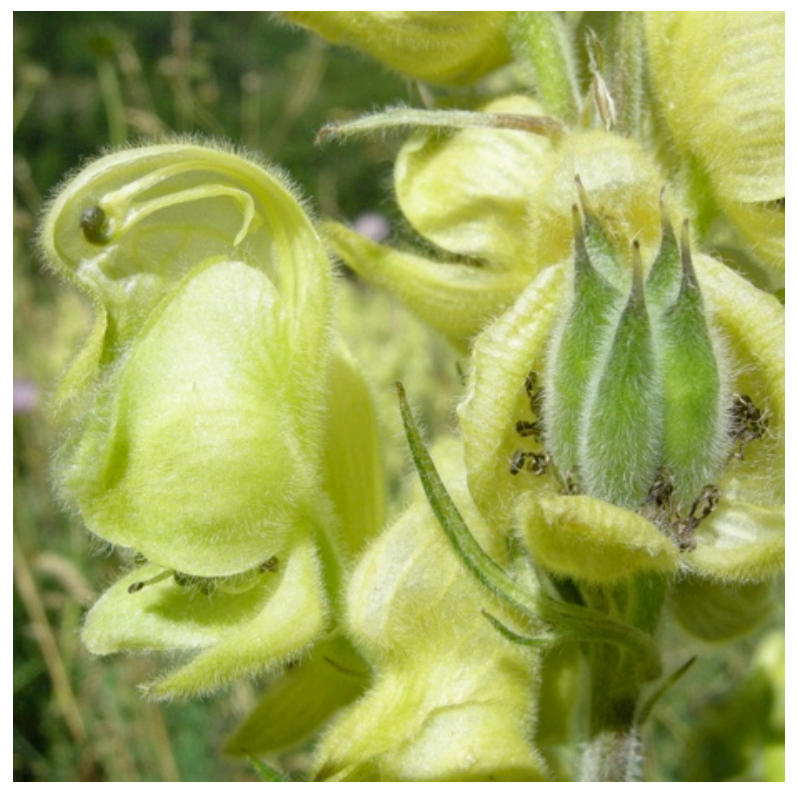

Figure 6. Disposition des nectaires chez Aconitum anthora (Ranunculaceae) (Photo G Mahé)

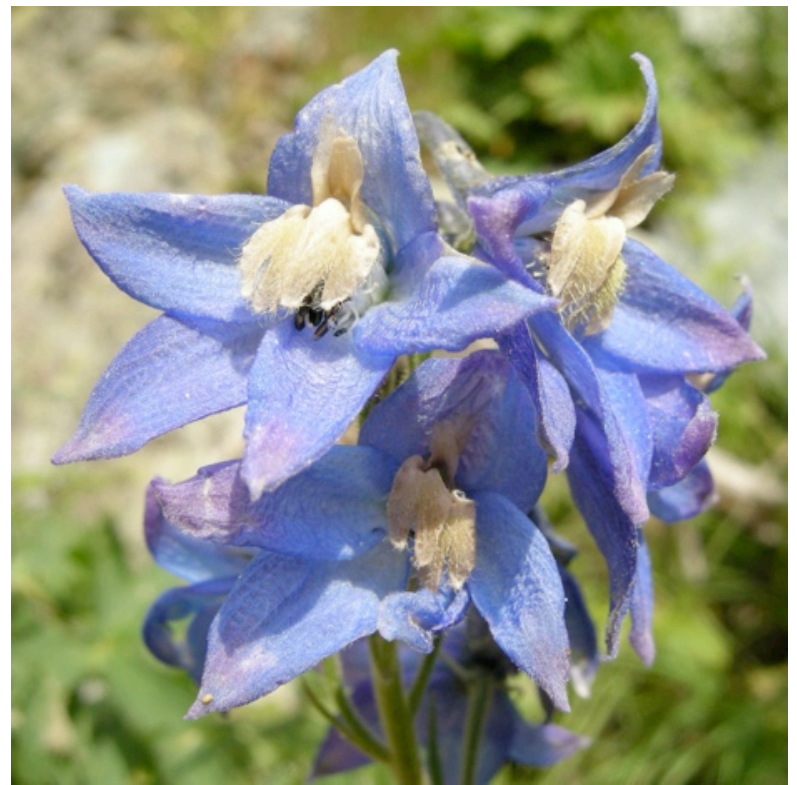

Figure 8. Détail d'une inflorescence de Delphinium dubium (Ranunculaceae) à pétales blancs (Photo G Mahé)

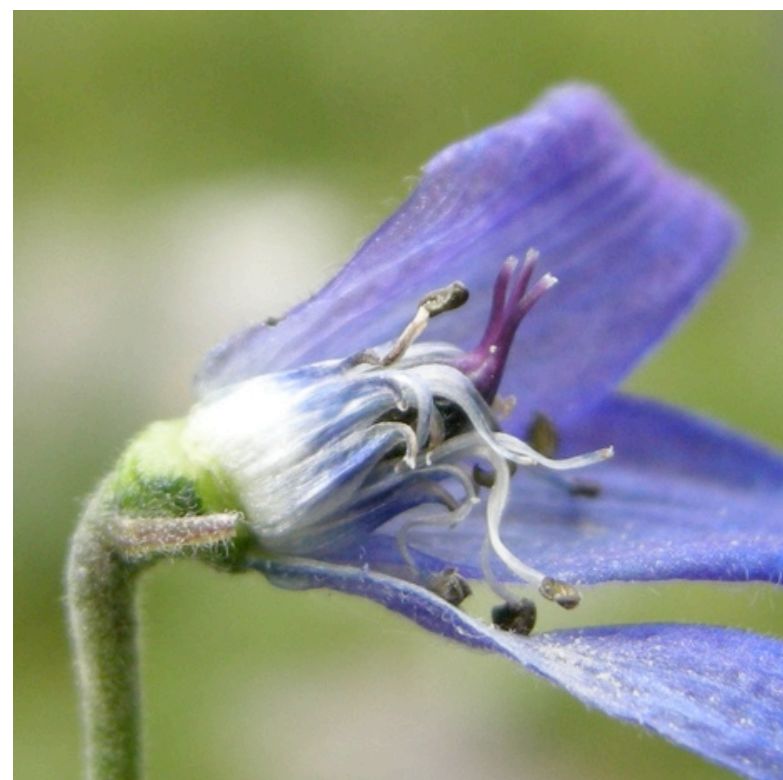

Figure 10. Redressement des styles du pistil chez Delphinium dubium (Ranunculaceae) (Photo G Mahé 


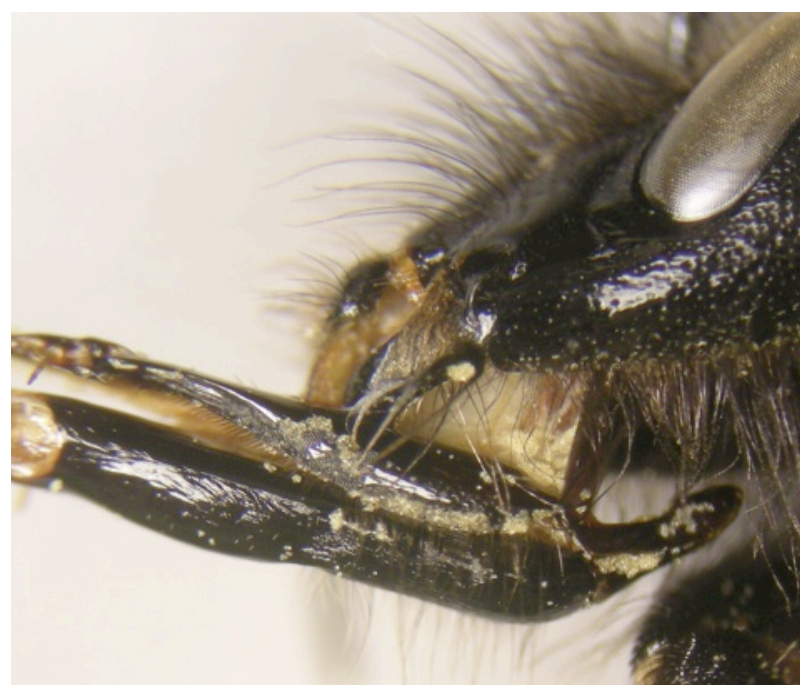

Figure 11. Proboscis de Bombus gerstaeckeri ayant visité des inflorescences de Delphinium dubium (Ranunculaceae) (Photo G Mahé)

B. inexspectatus. C'est la première fois que je voyais ce bourdon rare décrit récemment (Tkalcû 1963 \& 1965), et dont on sait si peu de chose encore aujourd'hui. Yarrow (1970) a suggéré que cette espèce parasitait probablement les nids de B. ruderarius ce que les observations de Müller (2006) semblent corroborer, mais d'autres observations seraient nécessaires pour confirmer cette hypothèse et préciser l'éthologie de cette espèce. $B$. inexspectatus ressemble beaucoup à $B$. ruderarius montanus (Figure 13) mais plusieurs critères permettent cependant de l'en distinguer. Chez la femelle l'un des caractères les plus remarquables est la forme très élargie du fossé labral (Figure 14). Chez le mâle la volselle possède une dent large et courte (Figure 15) alors qu'elle est beaucoup plus allongée chez $B$. ruderarius. D'autres critères tels que la pilosité, les micro-sculptures du corps et la longueur du troisième article des antennes sont venues conforter mes déterminations.

\section{Bombus brodmannicus}

Cerinthe minor auriculata (TEN.) ROUY est une plante assez fréquente dans le Queyras. J'espérais donc y rencontrer B. brodmannicus, le bourdon spécialisé sur les cerinthes. Malheureusement les fleurs de cette plante

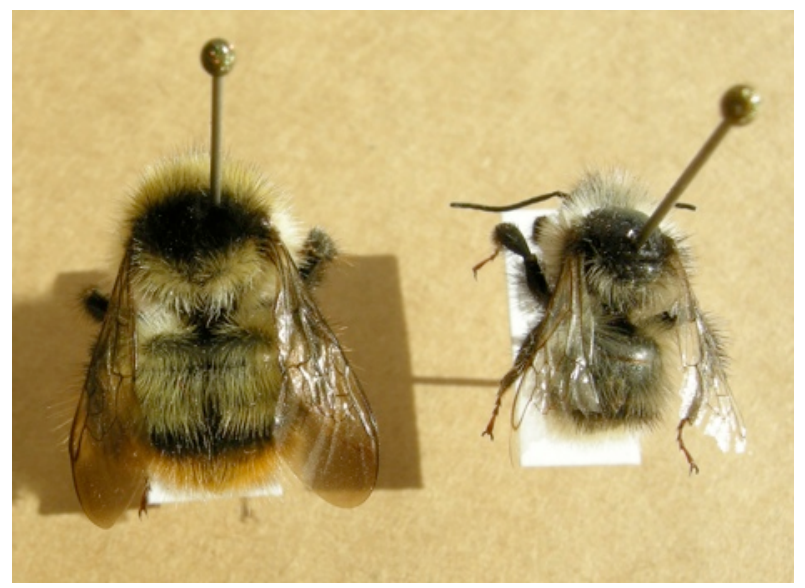

Figure 13. Bombus inexspectatus (Hymenoptera, Apidae), femelle (à gauche) et mâle (à droite) (Photo G Mahé)

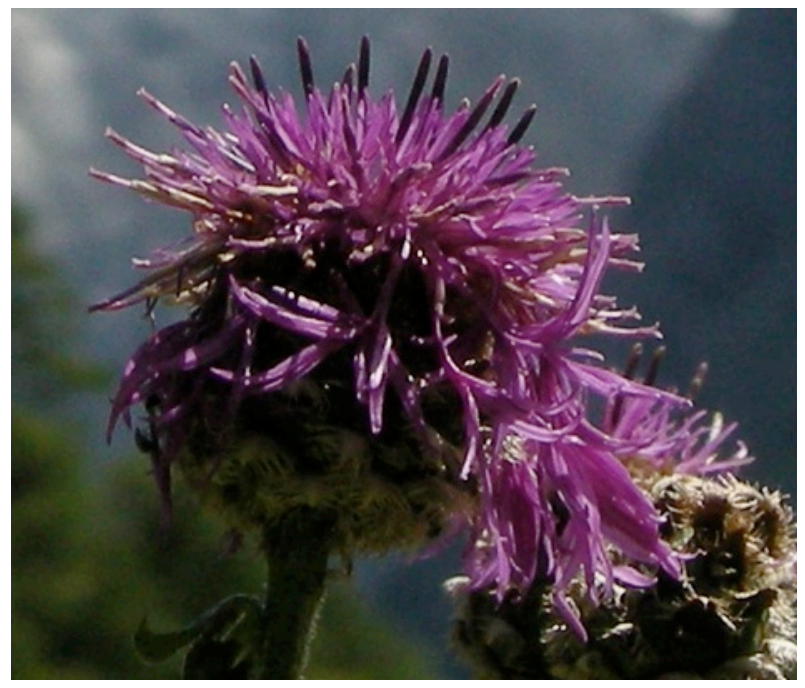

Figure 12. Détail d'une inflorescence de Centaurea sp. (Asteraceae) (Photo G Mahé)

sont généralement fanées en août. Cependant le 4 août 2006 à Sainte-Catherine (Haute-Alpes, Vars; WGS84 $\left.44,602^{\circ} \mathrm{N} 6,699^{\circ} \mathrm{E} ; 1920 \mathrm{~m}\right)$ j'avais capturé quatre ouvrières de ce bourdon butinant Stachys recta, ce qui montrait que $B$. brodmannicus pouvait se reporter sur d'autres plantes après la floraison des cerinthes. Je ne désespérais donc pas de voir encore cette année ce bourdon avec son pelage blanc-neige si caractéristique. Toute la semaine je l'ai cherché en scrutant attentivement les stations à Stachys, en vain. Finalement le 16 août 2007 près de la Chapelle de la Montette (Hautes-Alpes, Abriès ; WGS84 44,838 ${ }^{\circ} \mathrm{N}$ 6,945 ${ }^{\circ} \mathrm{E}$; $1960 \mathrm{~m})$ je trouve une station de plusieurs pieds de $C$. minor auriculata encore en fleur résultant semble-t-il d'un regain après pâturage. Je décide de m’y arrêter quelques instants pour noter les bourdons qui butinent cette plante. Je capture ainsi plusieurs ouvrières de $B$. pratorum, B. wurfleinii et B. ruderarius. Quand soudain à $15 \mathrm{~h} 30$ j'aperçois une superbe reine butinant les cerinthes (Figure 16). Il est difficile de décrire l'émotion que je ressens quand dans ce cadre grandiose du Parc naturel du Queyras, je trouve enfin l'animal remarquable que j’ai cherché plusieurs jours. Cela restera parmi les meilleurs souvenirs de mes vacances 2007.

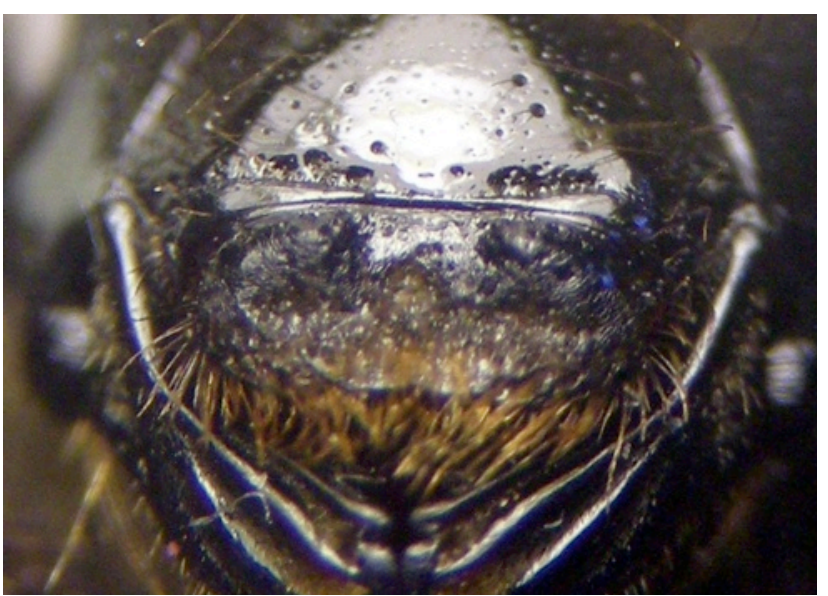

Figure 14. Détail du fossé labral d'une femelle de Bombus inexspectatus (Hymenoptera, Apidae) (Photo G Mahé) 


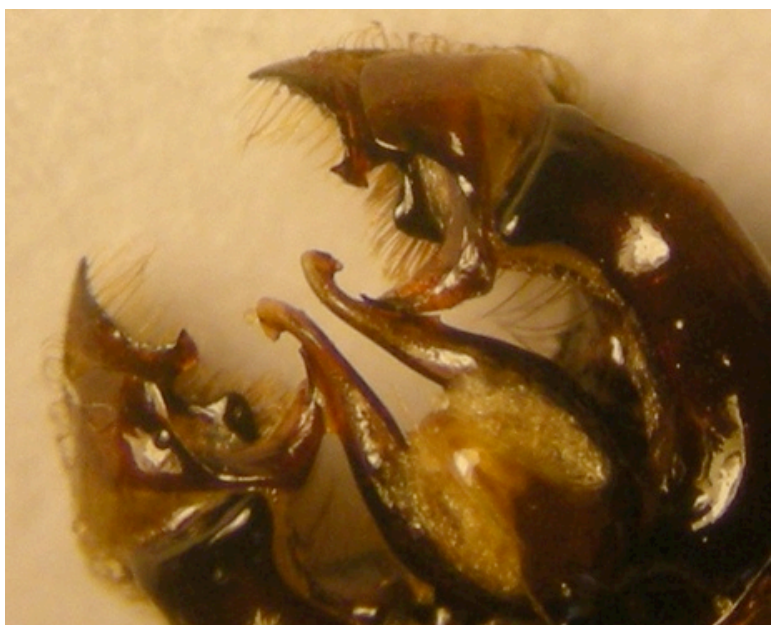

Figure 15. Détail des genitalia d'un mâle de Bombus inexspectatus (Hymenoptera, Apidae) (Photo G Mahé)

\section{L'accouplement de Bombus mendax}

Au moment de quitter les Alpes, je décide encore de faire une halte au col d'Izoard où j'avais observé le 30 juillet 2006 une forte population de B. mendax. Ce bourdon de haute altitude est assez commun dans le Queyras au dessus de 1800m (Rasmont 1988). Je l'y ai capturé en de nombreux points. Cependant le 17 août 2007, l'état de floraison au col est trop avancé, et je dois prospecter un peu les alentours avant de trouver aux Chalet de l'Izoard (Haute-Alpes, Cervières; WGS84 $44,830^{\circ} \mathrm{N} 6,715^{\circ} \mathrm{E} ; 2200 \mathrm{~m}$ ) une magnifique pelouse alpine richement fleurie. Je capture là diverses espèces tels que $B$. sichelii, B. monticola, B. subterraneus, B. hortorum, $B$. mesomelas, et $B$. rupestris. Mais ce qui me marque le plus c'est la présence de nombreuses reines de $B$. mendax qui butinent les fleurs du genre Cirsium, Trifolium et Gentianella. A un moment donné un bourdonnement inhabituel attire mon attention. Cela provient d'un mâle posé au sol qui vibre en continu à une dizaine de centimètres d'une reine également posée au sol. Tout en vibrant il se rapproche de la femelle qu'il chevauche, puis aussitôt les deux bourdons s'envolent accouplés. Le tout n'a duré que quelques secondes. J'ai bien essayé de les suivre mais malheureusement je n'ai pas pu les rattraper pour les capturer afin de les identifier formellement. Cependant étant donné la couleur de leur pelage, la liste et l'abondance de chaque espèce que j'ai trouvée sur le site, il est probable que j'ai assisté à un accouplement de $B$. mendax. Le comportement nuptial de cette espèce a été décrit par quelques auteurs (Haas, 1976). Bombus confusus SCHENCK et B. mendax sont les seuls bourdons de France qui attendent le passage des femelles à l'affût à partir d'un point fixe d'où ils chassent les intrus de toutes sortes qui s'aventurent sur leur "territoire". Mais l'accouplement ne semble jamais avoir été décrit ni chez B. mendax, ni chez B. confusus. Le col d'Izoard est un excellent site où de telles observations devraient être possibles. J'y reviendrai à l'occasion de prochaines vacances.

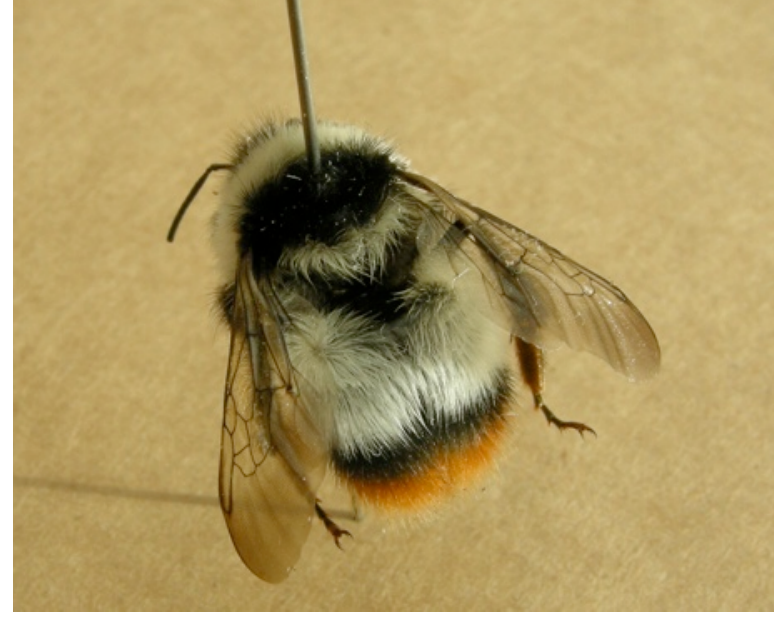

Figure 16. Reine de Bombus brodmannicus (Hymenoptera, Apidae) (Photo G Mahé)

\section{Conclusion}

Dans le Parc Naturel Régional du Queyras, il existe une belle diversité de milieux avec des pelouses alpines pâturées, des prairies de fauche, et aussi des milieux rocailleux qui sont d'une grande richesse floristique et qui permettent à des espèces rares de bourdons de s'y maintenir. Puisse ce type de paysage être conservé le plus longtemps possible.

\section{Remerciements}

Je remercie Pierre Rasmont (Université de Mons-Hainaut, Belgique) qui m'a transmis les documents utiles à la détermination de $B$. inexspectatus et qui a accepté de relire cet article.

\section{Références bibliographiques}

Haas A, 1976. Paarungsverhalten und Nestbau der alpin en Hummelart Bombus mendax (Hymenoptera: Apidae). Entomologica Germanica 3(3): 248-259.

Löken A, 1950. Bumble Bees in relation to Aconitum septentrionale in Western Norway (Eidfjord). Norsk Entomologisk Tidsskrift 8: $1-16$

Mahé G, 2007. Observations de Bombus gerstaeckeri MORAWITZ (Hymenoptera, Apidae) butinant Dephinium dubium (ROUY et Fouc.) PAWL. (Ranunculaceae) dans le Massif des Ecrins (Hautes-Alpes, France). OSMIA 1: 16-19

Müller A, 2006. A scientific note on Bombus inexspectatus (TKALCU, 1963): evidence for a social parasitic mode of life. Apidologie 37: 408-409.

Rasmont P, 1988. Monographie écologique et biogéographique des bourdons de France et de Belgique. Thèse de doctorat, Faculté des Sciences agronomiques de l'Etat, Gembloux, $309+$ LXII pp.

Rousseau S, 1994. Les relations coadaptatives des Aconits (Ranunculaceae, Aconitum L.) de Belgique et des Pyrénées avec leurs bourdons pollinisateurs (Hymenoptera, Apidae, Bombinae). Mémoire en vue de l'obtention du diplôme de Licencié en Sciences Zoologiques, Université de Mons-Hainaut, 53 + XXII pp.

Tkalcu B, 1963. Eine neue Hummel-Art der Gattung Agrobombus VOGT aus dem Alpengebiet (Hymenoptera, Apoidea). Casopis Ceskoslovenske Spolecnosti Entomologcké 60: 183-196.

Tkalcu B, 1965. Uber Agrobombus inexpectatus TKALCU (Hymenoptera, Apoidea, Bombinae). Reichenbachia 5: 225-230.

Utelli AB \& Roy BA, 2000. Pollinator abundance and behavior on Aconitum lycoctonum (Ranunculaceae) : an analysis of the quantity and quality components of pollination. Oikos 89: 461-470.

Yarrow IHH, 1970. Is Bombus inexspectatus (Tkalcu) a workerless obligate parasite? (Hymenoptera, Apidae). Insectes Sociaux 17: 95-112.
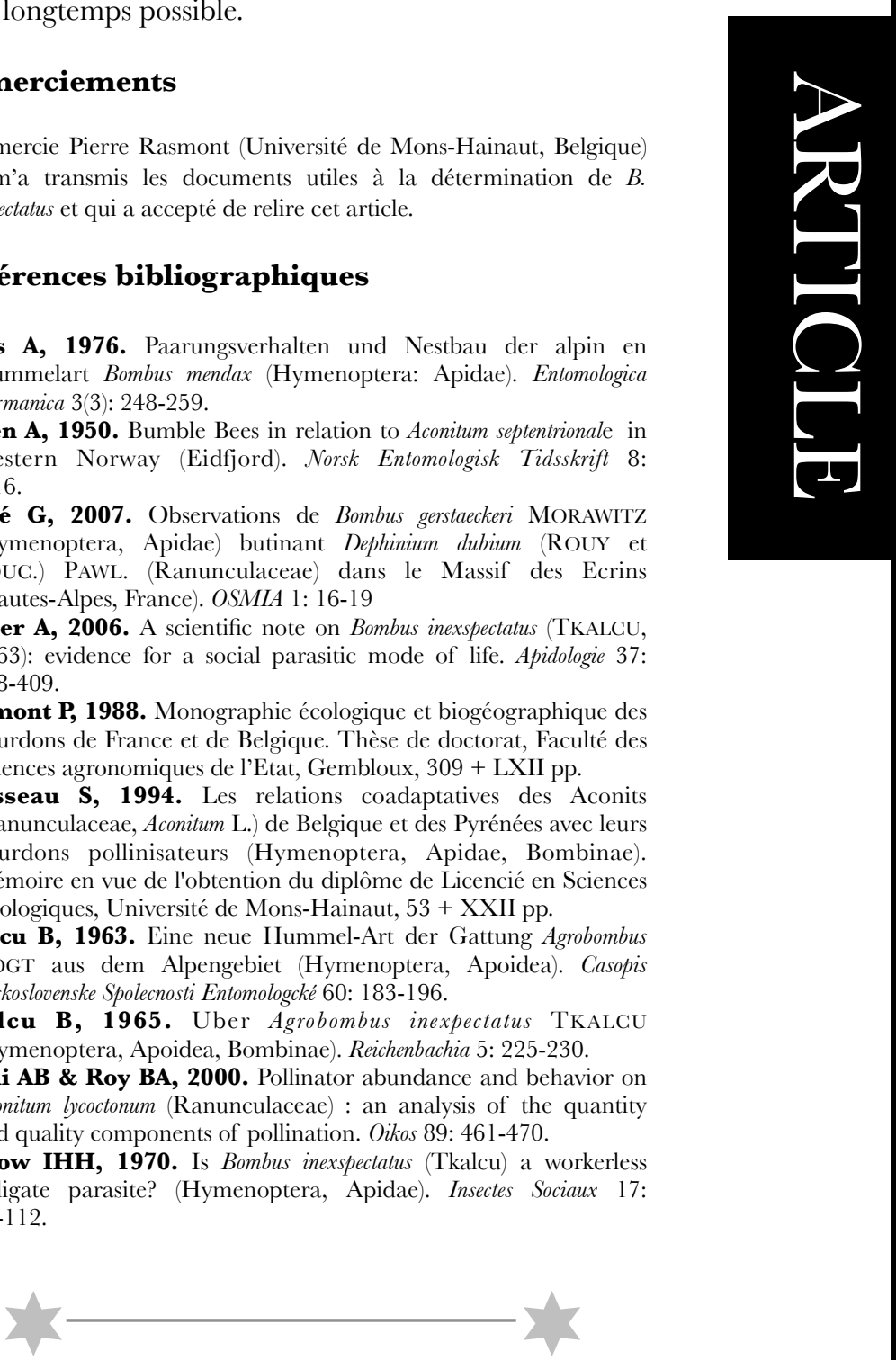